\title{
Transplantation of bone marrow-derived endothelial progenitor cells attenuates cerebral ischemia and reperfusion injury by inhibiting neuronal apoptosis, oxidative stress and nuclear factor- $\mathrm{kB}$ expression
}

\author{
JING QIU ${ }^{1,2^{*}}$, WEI LI ${ }^{3 *}$, SHUHANG FENG ${ }^{2}$, MIN WANG $^{2}$ and ZHIYI HE ${ }^{1}$ \\ ${ }^{1}$ Department of Neurology, The First Affiliated Hospital of China Medical University, Shenyang, Liaoning 110001; \\ ${ }^{2}$ Department of Neurology, The 463 Hospital of Chinese PLA, Shenyang, Liaoning 110042; ${ }^{3}$ Department of \\ Neurology, The General Hospital of Shenyang Military Region, Shenyang, Liaoning 110016, P.R. China
}

Received August 30, 2012; Accepted October 26, 2012

DOI: $10.3892 /$ ijmm.2012.1180

\begin{abstract}
The aim of the present study was to investigate the neuroprotective effects of bone marrow-derived endothelial progenitor cell (EPC) transplantation against cerebral ischemia/reperfusion (I/R) injury in rats and to delineate the possible underlying mechanisms. Cerebral I/R injury was established by $2 \mathrm{~h}$ of middle cerebral artery occlusion (MCAO) followed by reperfusion for $24 \mathrm{~h}$. EPCs were isolated from bone marrow of the donor rats, grown in conditioned medium, and characterized by flow cytometry analysis of several surface markers. Labeled EPCs $\left(10^{6}\right.$ cells $)$ were infused into rats at the onset of reperfusion and $12 \mathrm{~h}$ after reperfusion via the tail vein. Infarct volume was assessed at $24 \mathrm{~h}$ after reperfusion by using triphenyltetrazolium chloride (TTC) staining. The expression of cell apoptosisrelated proteins including Bcl-2 and Bax was determined by western blot analysis, and the activity of caspase- 3 was also measured. We evaluated the activities of some antioxidative enzymes, such as superoxide dismutase (SOD), glutathione peroxidase (GSH-PX), the non-enzymatic scavenger glutathione (GSH) and detected the content of malondialdehyde
\end{abstract}

Correspondence to: Dr Zhiyi He, Department of Neurology, The First Affiliated Hospital of China Medical University, 155 North Nanjing Street, Shenyang, Liaoning 110001, P.R. China

E-mail: cmulhhezhiyi@163.com

${ }^{*}$ Contributed equally

Abbreviations: EPC, endothelial progenitor cell; I/R, ischemia/ reperfusion; MCAO, middle cerebral artery occlusion; TTC, triphenyltetrazolium chloride; SOD, superoxide dismutase; GSH-PX, glutathione peroxidase; GSH, glutathione; MDA, malondialdehyde; $\mathrm{NF}-\kappa \mathrm{B}$, nuclear factor- $\kappa \mathrm{B}$

Key words: endothelial progenitor cell, cerebral ischemia and reperfusion injury, oxidative stress, apoptosis, nuclear factor- $\kappa \mathrm{B}$
(MDA) in the ischemic penumbra. Moreover, the expression of nuclear factor- $\mathrm{\kappa B}(\mathrm{NF}-\mathrm{\kappa B})$ in the ischemic regions of rats was examined by immunohistochemical staining and western blot analysis. The results showed that transplantation of EPCs significantly reduced the cerebral infarct volume, decreased caspase-3 activity, upregulated Bcl-2 expression, and downregulated the expression of Bax and NF- $\mathrm{KB}$. Furthermore, reduced levels of MDA, significantly elevated activities of SOD and GSH as well as GSH-PX were also found in I/R rats transplanted with EPCs. Collectively, our data demonstrated that transplantation of bone marrow-derived EPCs exerts potent neuroprotective functions against cerebral I/R injury in rats, and the protective effects may be associated with its antioxidative and anti-apoptotic properties.

\section{Introduction}

Stroke is the second most common cause of mortality and the leading cause of severe long-term disability in adults worldwide, affecting approximately 15 million people each year $(1,2)$. Ischemic stroke accounts for approximately $80 \%$ of all strokes, and occurs when a thrombus or embolism blocks a major cerebral blood vessel or its branches $(3,4)$. This blockage eventually leads to serious pathological changes and, possibly, to permanent impairment of brain functions (4). To date, thrombolysis with intravenous tissue plasminogen activator (t-PA) is the only approved therapy for patients with acute ischemic stroke, but only $1-2 \%$ of patients are eligible for this thrombolytic therapy primarily due to a narrow time window for administration $(5,6)$. Thus, there is an urgent need to develop novel therapeutic options for patients with ischemic stroke.

Endothelial progenitor cells (EPCs) are a specific subpopulation of hematopoietic stem cells capable of differentiating into mature endothelial cells (7). EPCs are produced in the bone marrow and mobilized into the peripheral circulation where they repair the damaged endothelium and promote the formation of new blood vessels (8). On the basis of their endothelial regenerative potential, there has been a growing interest in EPCs as novel biomarkers and potential therapeutic 
agents for a variety of pathological conditions, particularly cardiovascular diseases (9-11). Ohta et al (12) reported that administration of ex vivo-expanded bone marrow-derived EPCs reduces infarct volume and neurological deficits in acute focal brain ischemia and reperfusion (I/R) injury, possible by attenuation of endothelial dysfunction. However, EPC studies in stroke have been limited (13), and the molecular mechanisms underlying the neuroprotective effects of bone marrow-derived EPCs have not been fully elucidated.

Apoptosis is a major cellular mechanism responsible for cell death in the pathological processes after cerebral I/R injury (14). Oxidative stress has been reported to occur in response to cerebral I/R injury and is thought to be one of the major contributors to neuronal death (15). Nuclear factor- $\kappa \mathrm{B}$ $(\mathrm{NF}-\kappa \mathrm{B})$, an important transcriptional factor, plays a critical role in regulating cellular responses to oxidative stress (16). In addition, previous studies have demonstrated that $\mathrm{NF}-\kappa \mathrm{B}$ is activated during cerebral I/R injury (17), and the inhibition of $\mathrm{NF}-\kappa \mathrm{B}$ exhibits potent neuroprotective effects during cerebral ischemia (18). Based on these findings, we hypothesized that transplantation of bone marrow-derived EPCs may attenuate cerebral I/R injury through the inhibition of neuronal apoptosis and oxidative stress.

In the present study, we used a rat model of middle cerebral artery occlusion (MCAO) to further investigate the neuroprotective effects of bone marrow-derived EPCs as well as the underlying mechanisms by focusing on neuronal apoptosis, oxidative stress, and NF- $\kappa \mathrm{B}$ expression.

\section{Materials and methods}

Animals. Male Sprague-Dawley rats, weighing 200-250 g, were obtained from the Experimental Animal Centre of China Medical University, Shenyang, China. The experimental protocol was reviewed and approved by the Institutional Animal Care and Use Committee of China Medical University. All animals were housed under diurnal lighting conditions $\left(22 \pm 3^{\circ} \mathrm{C}\right.$ and a $12 \mathrm{~h}$ light/dark cycle) with free access to standard laboratory chow and water.

Isolation and in vitro culture EPCs. Isolation and ex vivo expansion of EPCs were performed as previously described $(19,20)$. Briefly, femur and tibias were excised from the donor rats after they were anesthetized with chloral hydrate $(300 \mathrm{mg} / \mathrm{kg}$ ) and sacrificed by cervical dislocation. Total bone marrow cells were harvested aseptically by flushing femurs and tibias with phosphate buffered saline (PBS), and mononuclear cells were collected by density gradient centrifugation with Histopaque-1083 (Sigma-Aldrich, St. Louis, MO, USA). After washing twice in PBS, the obtained mononuclear cells were seeded on rat plasma fibronectin-coated dishes and maintained in EC basal medium-2 (EBM-2) supplemented with 5\% fetal bovine serum (FBS; Invitrogen, Carlsbad, CA, USA), antibiotics, and growth factors. Cells were cultured at $37^{\circ} \mathrm{C}$ in a humidified atmosphere with $5 \% \mathrm{CO}_{2}$. After 4 days of culture, the non-adherent cells were removed by washing with PBS, and fresh culture medium was applied. On the tenth day, the adherent cells were harvested by trypsinization, washed, and resuspended for transplantation or immunophenotypic characterization by flow cytometry.
Flow cytometry. To characterize the EPC population, flow cytometry was performed on freshly isolated cells (at Day 0) and on cells after 10 days of culture by using the antibodies against a panel of surface markers, including CD31, CD34, CD133 and fetal liver kinase-1 (Flk-1). Briefly, the cells were harvested, washed twice with PBS, and then incubated for $30 \mathrm{~min}$ at $4^{\circ} \mathrm{C}$ with mouse anti-rat antibodies for CD31 and CD34 (Santa Cruz Biotechnology, Inc., Santa Cruz, CA, USA) and rabbit anti-rat antibodies for CD133 and Flk-1 (Abnova, Taipei, Taiwan), followed by their corresponding fluorescein isothiocyanate (FITC)-labeled secondary antibodies. Finally, the samples were analyzed with a FACSCalibur cytometer (BD Biosciences, San Jose, CA, USA) and data were processed using the CellQuest software program.

EPC labeling for in vivo tracking. In order to track the fate of transplanted cells, EPCs after 10 days of culture were labeled with a lipophilic red fluorescent dye, CM-Dil (Invitrogen), according to the manufacturer's instructions. Thirty minutes before transplantation, EPCs were incubated with $2 \mu \mathrm{l} \mathrm{CM-Dil}$ $(1 \mathrm{mg} / \mathrm{ml})$ at $37^{\circ} \mathrm{C}$ and then resuspended in $500 \mu$ l culture medium for administration.

Experimental groups, rat model of cerebral $I / R$ injury and $E P C$ administration. Fifty-four rats were randomly divided into three groups as follows (18 rats in each group): the sham group, without MCAO and culture medium-treated; the I/R group, $2 \mathrm{~h}$ occlusion followed by $24 \mathrm{~h}$ reperfusion and culture medium-treated; the EPC group, with MCAO and EPC administration.

A rat model of cerebral I/R injury was established by MCAO as previously reported (21). In brief, after the rats were anesthetized with $10 \%$ chloral hydrate $(350 \mathrm{mg} / \mathrm{kg}$, i.p.), the right common carotid artery (CCA), internal carotid artery (ICA), and external carotid artery (ECA) were surgically exposed through a ventral middle line incision. A 4-0 nylon monofilament nylon suture with a heat-rounded tip was introduced into the ECA lumen and gently advanced into the ICA lumen until a slight resistance was encountered, thus to occlude the origin of the middle cerebral artery. Two hours later, the filament was carefully withdrawn to restore cerebral blood flow and the neck incision was sutured. The rectal temperature was maintained between 36.5 and $37.5^{\circ} \mathrm{C}$ by using a heat pad and a heat lamp throughout the surgical procedure. Shamoperated animals underwent the same surgical procedures but without inserting a filament.

Labeled EPCs $\left(10^{6}\right.$ cells in $500 \mu 1$ culture medium) were infused into the rats in the EPC group at the onset of reperfusion and $12 \mathrm{~h}$ after reperfusion via the tail vein. Rats in the sham and $\mathrm{I} / \mathrm{R}$ groups were injected in the same manner but only with an equal volume of fresh culture medium. After $24 \mathrm{~h}$ of reperfusion, all animals were deeply anesthetized with chloral hydrate $(300 \mathrm{mg} / \mathrm{kg})$ and sacrificed by decapitation.

Measurement of cerebral infarct volume. After decapitation, the brains $(n=6)$ were quickly collected, chilled in ice-cold saline for $5 \mathrm{~min}$, and sectioned coronally into eight, $1.5 \mathrm{~mm}$ slices. The slices were stained with $2 \%$ 2,3,5-triphenyltetrazolium chloride (TTC; Sigma-Aldrich) at $37^{\circ} \mathrm{C}$ for $20 \mathrm{~min}$, followed by fixation in $4 \%$ paraformaldehyde overnight. 
The normal tissues were stained dark red, while the infarct areas remain unstained (white). The stained slices were photographed with a digital camera, and the infarct area was quantified with an image analysis system (Image-Pro Plus). The infarct volumes were presented as percentages of the total brain volume (22).

Measurement of superoxide dismutase (SOD), glutathione $(G S H)$, glutathione peroxidase (GSH-PX), malondialdehyde (MDA) and caspase-3 activities. Antioxidative enzyme activities including SOD, GSH-PX, GSH and MDA in the hippocampus homogenate were measured by using commercially available kits (Jiancheng Institute of Bioengineering, Nanjing, China), according to the recommended protocols. SOD activity was determined by monitoring its ability to inhibit nucleotide oxidation. Results were reported as U/mg protein. A GSH-PX unit activity was defined as the amount of enzyme required to convert $1 \mu \mathrm{mol}$ of reduced GSH to the oxidized form of glutathione $\mathrm{GSH}$ in the presence of $\mathrm{H}_{2} \mathrm{O}_{2} / \mathrm{min}$. MDA content was determined based on its activity to react with thiobarbituric acid and is expressed as nmol/mg protein. The activity of caspase- 3 in the hippocampus homogenate was assayed by using a caspase- 3 activity kit (Beyotime Institute of Biotechnology, Haimen, P.R. China) following the manufacturer's instructions. Caspase-3 activity was estimated by measuring Ac-DEVD-pNA reacting substances at the wavelength of $405 \mathrm{~nm}$.

Immunohistochemistry. The brains were immersed in $10 \%$ buffered formalin overnight, embedded in paraffin, and sectioned with a thickness of $5 \mu \mathrm{m}$. After dewaxing with xylene and rehydrating through descending ethanol, the sections were subjected to heat-mediated antigen retrieval in citrate buffer solution (pH 6.0). Subsequently, endogenous peroxidase activity was inactivated using $3 \%(\mathrm{v} / \mathrm{v})$ hydrogen peroxide at room temperature for $15 \mathrm{~min}$, and nonspecific binding of antibodies was blocked with $10 \%$ normal goat serum. The sections were then incubated overnight at $4^{\circ} \mathrm{C}$ in a humidifying chamber with a rabbit anti-rat NF- $\kappa$ B polyclonal antibody (1:200 diluted; Santa Cruz Biotechnology, Inc.). After rinsing in PBS, the corresponding secondary antibody [biotinylated goat anti-rabbit immunoglobulin G (IgG), 1:200 diluted; Zhongshan Golden Bridge Biotechnology, Beijing, China] was applied, followed by incubation for 30 min with streptavidin-horseradish peroxidase conjugate. The final immunoreactivity was visualized by using 3,3'-diaminobenzidine (DAB), and all sections were then counterstained with hematoxylin, dehydrated, and mounted. For negative controls, an isotype matched $\mathrm{IgG}$ was used instead of the primary antibody.

Western blot analysis. Total proteins were extracted from the ipsilateral ischemic cortex, and protein concentrations were quantified using bicinchoninic acid (BCA) protein assay kit (Beyotime Institute of Biotechnology). An equivalent amount of proteins was resolved on sodium dodecyl sulfate polyacrylamide gels (SDS-PAGE), and then electrotransferred onto polyvinylidene fluoride (PVDF) membranes (Millipore, Bedford, MA, USA) under $70 \mathrm{~V}$ constant voltage conditions for $90 \mathrm{~min}$. After blocking with $5 \%$ fat-free milk at room temperature for $2 \mathrm{~h}$, the membranes were immunoblotted overnight at $4^{\circ} \mathrm{C}$ with rabbit anti-Bcl-2, anti-Bax, (1:1,000 diluted; Abcam,

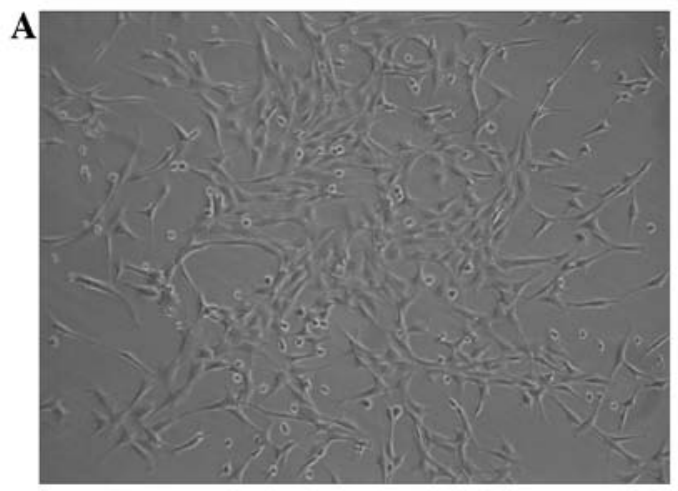

$\mathbf{B}$
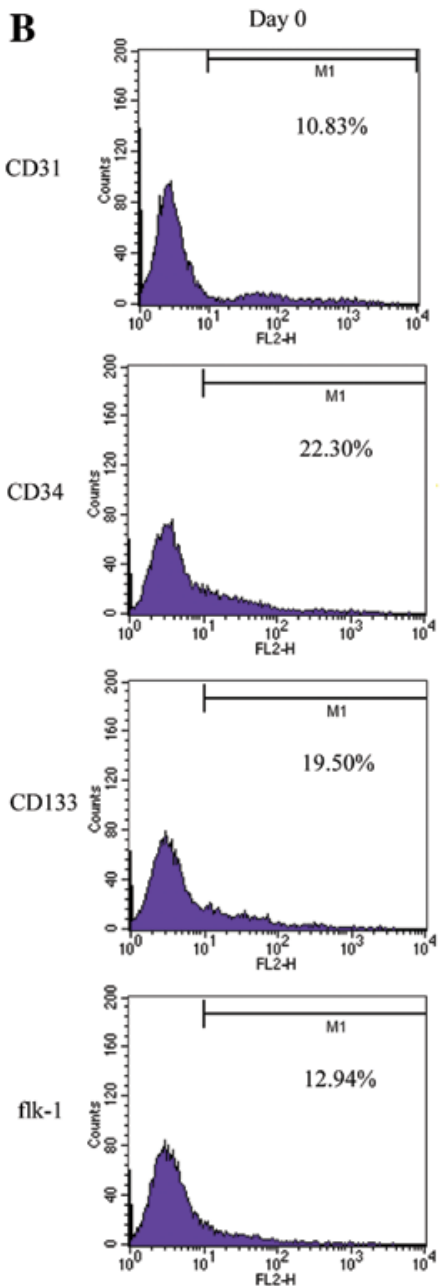
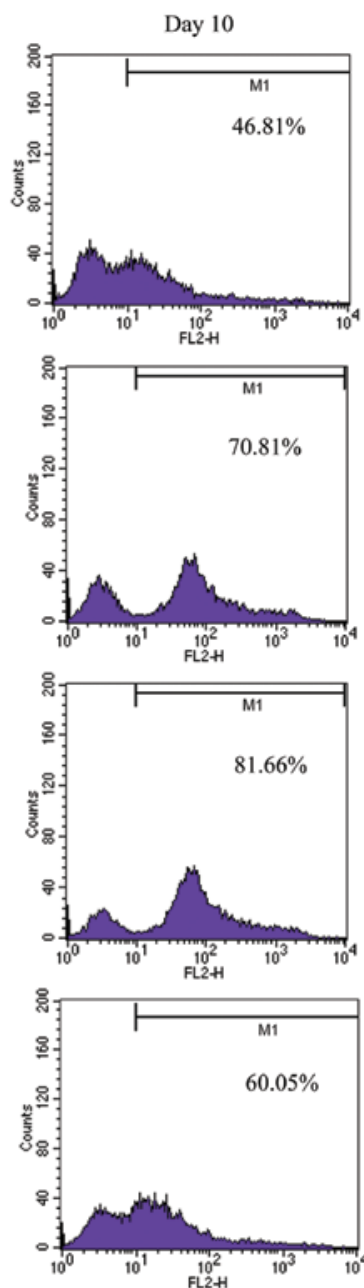

Figure 1. In vitro characteristics of bone marrow EPCs after 10-day culture. (A) After 10 days of culture, spindle-shaped morphology or cobblestone-like appearance typical of endothelial cells were observed under high-power field. (B) Flow cytometry analysis of the isolated rat EPCs at Day 0 and 10 days after culture. The percentage of each type of marker-positive cells is shown in the graph.

Cambridge, MA, USA), or anti-NF- $\kappa$ B antibody $(1: 1,000$ diluted; Santa Cruz Biotechnology, Inc.), followed by horseradish peroxidase-conjugated secondary antibodies. Following extensive washing, immunoblots were visualized with an enhanced chemiluminescence (ECL) detection kit (Millipore). The intensity of the bands was quantified by densitometric analysis and normalized to the loading control $\beta$-actin. 

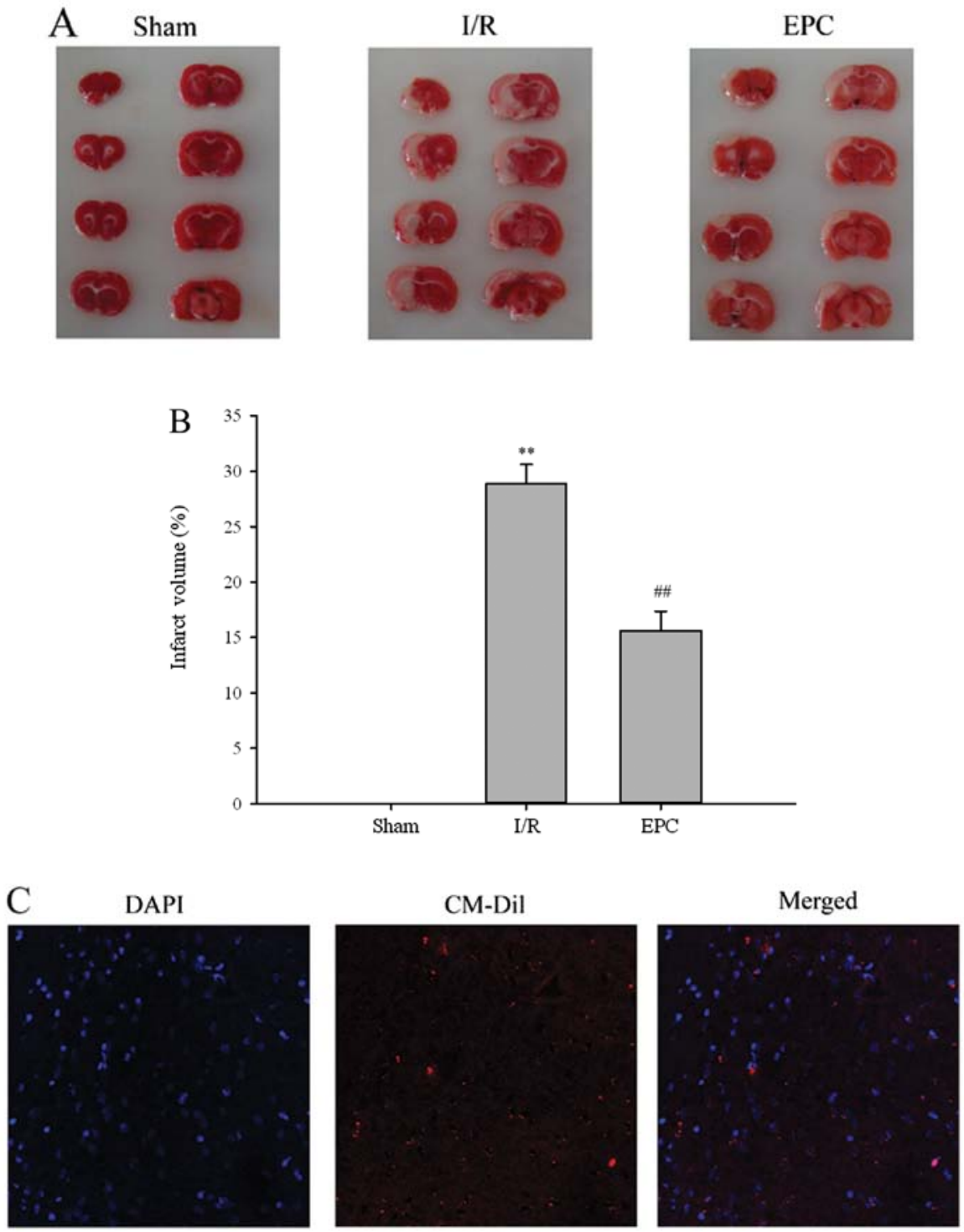

Figure 2. Neuroprotective effects of EPC administration on cerebral I/R injury in rats. (A) Representative coronal brain sections stained with $2 \%$ TTC from the sham, I/R and EPC groups after $2 \mathrm{~h} \mathrm{MCAO}$ and $24 \mathrm{~h}$ reperfusion. (B) Quantitative analysis of the infarct volume ( $\mathrm{n}=6,{ }^{* *} \mathrm{P}<0.01 \mathrm{vs}$. the sham group; ${ }^{\# \#} \mathrm{P}<0.01$ vs. the I/R group). (C) Identification of CM-Dil-stained EPCs (red fluorescence) in the hippocampus of ischemia hemisphere from the EPC group.

Statistical analysis. All data are expressed as the means \pm standard deviation (SD). Statistical analysis was performed using one-way ANOVA followed by the Bonferroni post-hoc test for individual comparisons between group means. Graphs were plotted and statistical calculations were carried out using SigmaPlot 12.0 (Systat Software, Inc., San Jose, CA, USA). A P-value $<0.05$ was considered to indicate statistically significant differences.

\section{Results}

In vitro characteristics of bone marrow EPCs after 10-day culture. After 10 days of culture in EC basal medium, the bone marrow-derived mononuclear cells exhibited spindleshaped morphology or cobblestone-like appearance typical of endothelial cells (Fig. 1A). To further characterize these cells, the stem cell markers (CD34 and CD133) and endothelial cell markers (CD31 and Flk-1) were analyzed by flow cytometry according to previous studies $(23,24)$. Compared with freshly isolated mononuclear cells, the percentages of cell population positive for CD34, CD133, CD31 and Flk-1 were increased from $22.30 \pm 2.56$ to $70.81 \pm 4.89 \%, 19.5 \pm 2.95$ to $81.66 \pm 5.33 \%$, $10.83 \pm 1.12$ to $46.81 \pm 3.54 \%$ and $12.94 \pm 1.87$ to $60.05 \pm 3.91 \%$, respectively (Fig. 1B). These cells were therefore confirmed as bone marrow-derived EPCs.

Effects of EPC transplantation on brain infarct volume. To investigate the neuroprotective effects of EPC transplantation on cerebral I/R injury, EPCs $\left(10^{6}\right.$ cells) were administered intravenously at the onset of reperfusion and $12 \mathrm{~h}$ after reperfusion, and infarct volumes were assessed at $24 \mathrm{~h}$ after reperfusion by TTC staining. As shown in Fig. 2A and B, no infarction was observed in sham-operated group animals. By contrast, the infarct volume was $28.9 \pm 1.8 \%$ after $2 \mathrm{~h} \mathrm{MCAO}$ and $24 \mathrm{~h}$ reperfusion in the I/R group. However, EPC transplantation significantly reduced cerebral infarct volumes, indicating neuroprotective effects of EPC transplantation against cerebral I/R injury. Moreover, under immunofluores- 

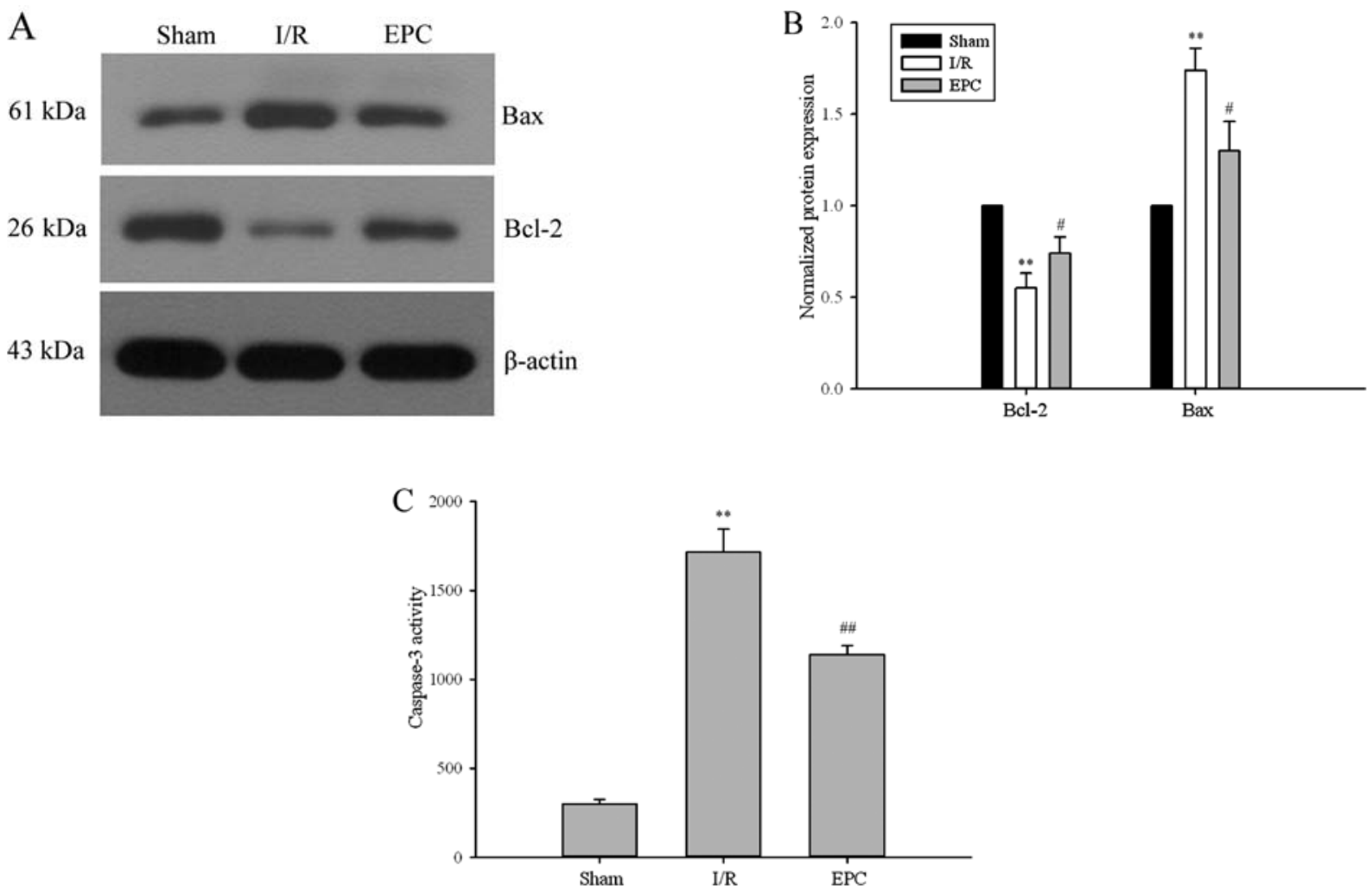

Figure 3. Effects of EPC transplantation on Bcl-2 and Bax expression and caspase-3 activity in ischemic penumbra cortex at $24 \mathrm{~h}$ after reperfusion. (A) Western blot analysis of Bcl-2 and Bax protein levels. Representative blots are presented and the protein size is expressed in $\mathrm{kDa}$. (B) Quantitative data are expressed as the intensity ratio of target proteins to $\beta$-actin. (C) The activity of caspase- 3 was assayed using a caspase- 3 activity kit. $\left(\mathrm{n}=6,{ }^{* *} \mathrm{P}<0.01 \mathrm{vs}\right.$. the sham group; ${ }^{\# \prime} \mathrm{P}<0.01,{ }^{\#} \mathrm{P}<0.05$ vs. the $\mathrm{I} / \mathrm{R}$ group).

cence microscopy, a significant number of CM-Dil-labeled EPCs showing red fluorescence adoptively transferred into the infarct area, suggesting that EPCs can migrate into the ischemic rat brain within $24 \mathrm{~h}$ after intravenous injection.

Effects of EPC transplantation on the expression of Bcl-2 and Bax proteins and caspase-3 activity in hippocampus after $I / R$ injury. To test if EPC transplantation exerts its neuroprotective effects against cerebral I/R injury via the inhibition of neuronal apoptosis, we detected the expression of $\mathrm{Bcl}-2$ and Bax protein in the ischemic penumbra of rats with $2 \mathrm{~h} \mathrm{MCAO}$ and $24 \mathrm{~h}$ reperfusion by western blot analysis. The results showed that cerebral I/R injury induced by MCAO caused significant upregulation of Bax and downregulation of Bcl-2 protein levels; however, these changes were remarkably attenuated by EPC transplantation $(\mathrm{P}<0.05, \mathrm{n}=6)$ (Fig. 3A and $\mathrm{B})$. We also measured the enzymatic activity of caspase-3, a reliable indicator for assessment of apoptosis. The activity of caspase- 3 in the ischemic penumbra of rats with $2 \mathrm{~h} \mathrm{MCAO}$ and $24 \mathrm{~h}$ reperfusion was significantly increased compared with the sham group, while the caspase- 3 activity was markedly reduced in the EPC group $(\mathrm{P}<0.05, \mathrm{n}=6)$ (Fig. $3 \mathrm{C})$. These findings suggest that EPC transplantation has the potential to protect hippocampal neurons against cerebral I/R injury-induced apoptosis.

Effects of EPC transplantation on the content of MDA and the activities of antioxidant enzymes in hippocampus after $I / R$ injury. To further determine whether the neuroprotective effects of EPC transplantation are associated with the inhibition of oxidative stress, we evaluated the content of MDA and the activities of antioxidant enzymes in the ischemic penumbra of rats with $2 \mathrm{~h}$ MCAO and $24 \mathrm{~h}$ reperfusion. As illustrated in Fig. 4A, the level of MDA, an index of lipid peroxidation, was significantly increased in culture medium-treated ischemic hippocampus from $0.61 \pm 0.09$ to $2.19 \pm 0.32 \mathrm{nmol} / \mathrm{mg}$ protein $(\mathrm{P}<0.01, \mathrm{n}=6)$. After administration of EPCs, the I/R-induced lipid peroxidation was evidently decreased to $1.37 \pm 0.17 \mathrm{nmol} / \mathrm{mg}$ protein $(\mathrm{P}<0.01, \mathrm{n}=6)$ compared to the $\mathrm{I} / \mathrm{R}$ group. Additionally, the activities of antioxidant enzymes including GSH, GSH-PX and SOD in the I/R group were remarkably diminished compared to the sham group $(\mathrm{P}<0.01, \mathrm{n}=6)$. However, EPC transplantation resulted in enhanced activities compared to the $\mathrm{I} / \mathrm{R}$ group $(\mathrm{P}<0.05, \mathrm{n}=6)$. Collectively, our data indicate that administration of EPCs exerts neuroprotective effects via its ability to inhibit oxidative stress.

Effects of EPC transplantation on $N F-\kappa B$ expression in hippocampus after I/R injury. Next, we analyzed the effects of EPC transplantation on NF- $\mathrm{KB}$ expression in hippocampus after I/R injury by immunohistochemistry and western blot analysis. Few NF- $\kappa B$ positive cells were observed in the hippocampus of sham-operated animals (Fig. 5A). At $24 \mathrm{~h}$ after reperfusion, an intense staining of NF- $\mathrm{kB}$ was found in the nucleus in the ischemic hippocampus. However, EPC implantation significantly attenuated the rise of brain I/R-induced NF- $\kappa \mathrm{B}$ expression. Meanwhile, changes observed by western blot analysis were in 

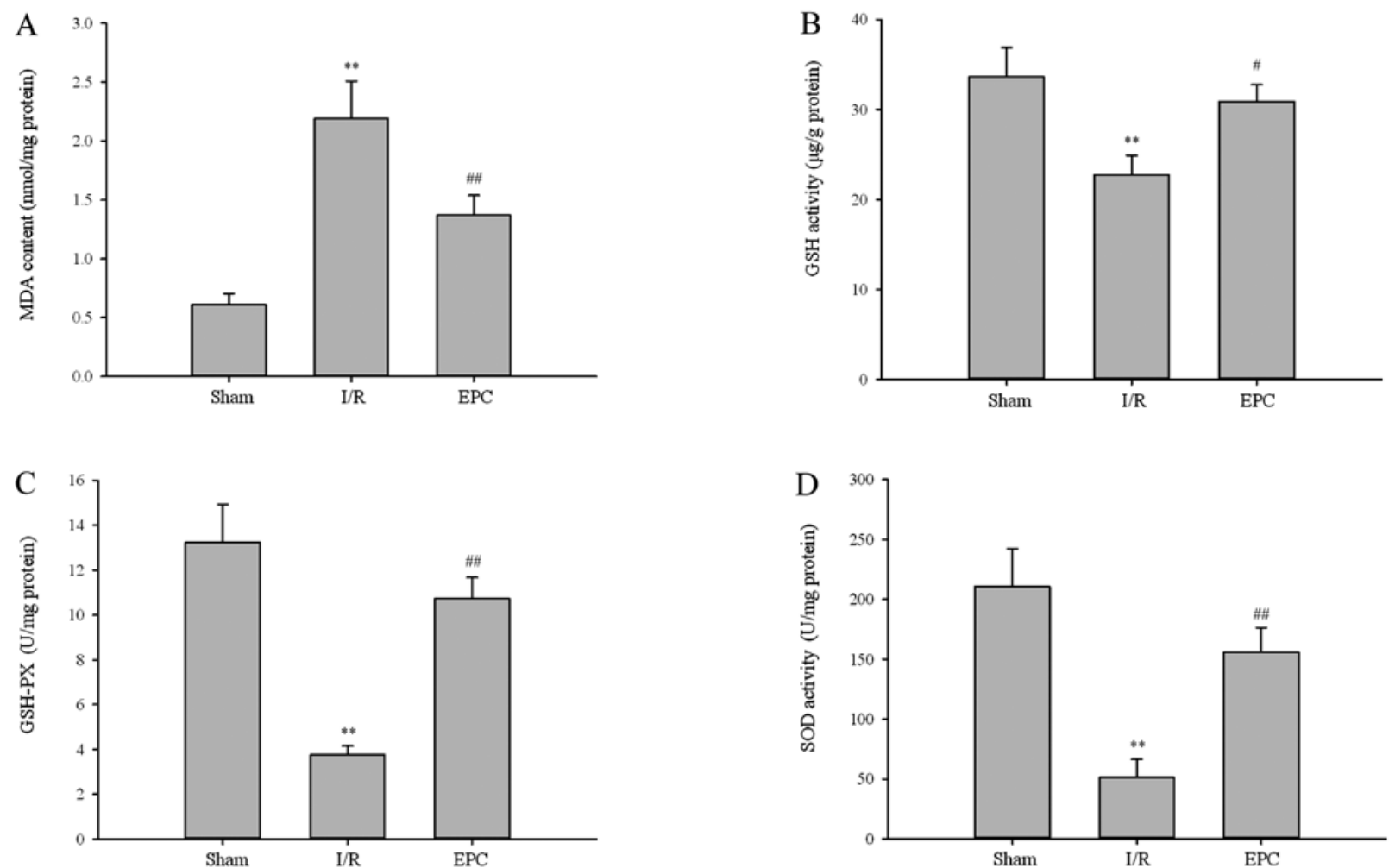

Figure 4. Effects of EPC transplantation on the content of MDA and the activities of antioxidant enzymes in ischemic penumbra cortex at $24 \mathrm{~h}$ after reperfusion. The effects of EPC transplantation on (A) MDA, (B) GSH, (C) GSH-PX and (D) SOD activities in hippocampus, respectively, are shown. (n=6, ** $\mathrm{P}<0.01 \mathrm{vs.}$ the sham group; ${ }^{\# \#} \mathrm{P}<0.01,{ }^{\#} \mathrm{P}<0.05$ vs. the $\mathrm{I} / \mathrm{R}$ group).
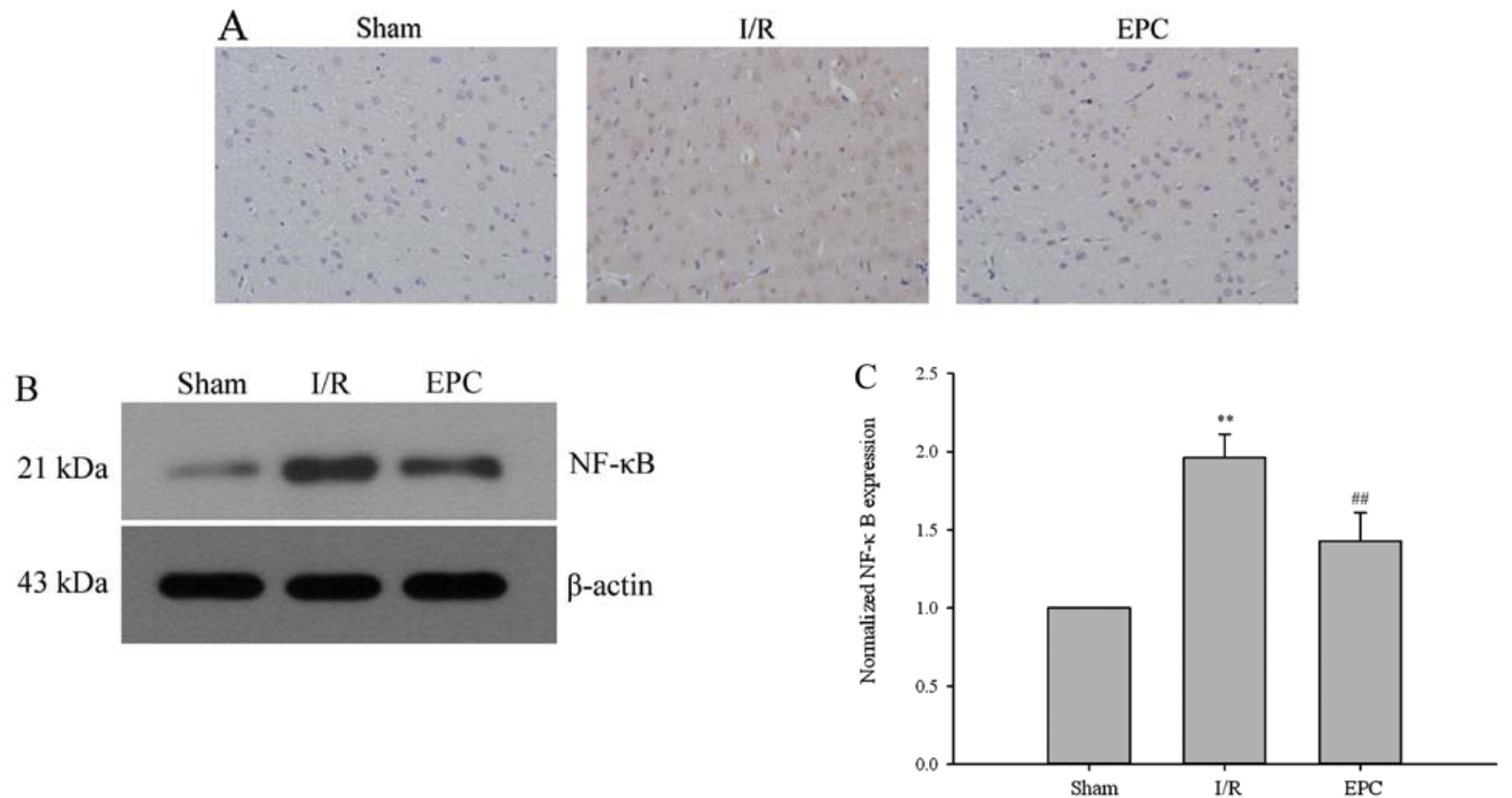

Figure 5. Effects of EPC transplantation on the expression of NF- $\mathrm{KB}$ in ischemic regions of rat cortex induced by $2 \mathrm{~h}$ MCAO and $24 \mathrm{~h}$ reperfusion as shown by (A) immunohistochemical staining, and (B) western blot analysis. Representative blots of three independent experiments are shown. (C) Densitometric values were normalized by $\beta$-actin levels. $\left(\mathrm{n}=6,{ }^{* *} \mathrm{P}<0.01\right.$ vs. the sham group; ${ }^{\# \#} \mathrm{P}<0.01$ vs. the $\mathrm{I} / \mathrm{R}$ group).

accordance with the observations in the immunohistochemical staining (Fig. 5B and C). These findings demonstrate that EPC transplantation is capable of inhibiting brain I/R injuryinduced NF- $\mathrm{KB}$ activation.

\section{Discussion}

The intraluminal suture method of MCAO in rodents is a well-characterized and classical animal model to mimic the 
pathological features of human cerebral I/R injury and has been widely used in pharmacological and molecular studies of brain I/R injury (4). In the present study, we used the MCAO animal model and demonstrated that EPC transplantation protected against cerebral $\mathrm{I} / \mathrm{R}$ injury by reducing infarct volume, which is associated with the inhibition of neuronal apoptosis, oxidative stress and NF- $\mathrm{KB}$ expression.

Despite extensive research efforts to standardize the definitions of EPCs, the exact definition and molecular characterization of EPCs is an ongoing debate (11). EPCs were initially identified by Asahara et al (7) as CD34-positive mononuclear cells from adult peripheral blood. It is now known that EPCs migrate from the bone marrow to the peripheral blood as circulating EPCs, where they express CD133, CD34 and Flk-1 (25). Currently, the most commonly accepted definition of EPCs includes co-expression of CD31, CD133, CD34 and Flk-1 (9). In this study, the molecular characteristics of EPCs isolated and expanded from bone marrow were similar to those described in previous reports (10). EPC administration significantly attenuated cerebral I/R injury, and fluorescent-labeled EPCs were observed in the hippocampus of the ischemia-affected hemisphere. These results are in agreement with the findings from Ohta et al (12). Notably, the discovery rate of EPCs was relatively low in the parenchyma of the ischemic hemisphere. Our data suggest that EPCs may exert neuroprotective benefits via their paracrine effective in addition to neovascularization.

A large body of evidence suggests that neuronal apoptosis represents an important mechanism in the pathophysiological mode of cell death in ischemic brain injury (14). Caspase-3 plays a critical role in apoptotic cell death, and the inhibition of caspase-3 has been shown to ameliorate cerebral ischemic injury (26). In the current study, we consistently found a marked increase in caspase-3 activity in the ischemic rat penumbra, which could be effectively dampened by administration of EPCs. Bcl-2 is an anti-apoptotic and Bax is a pro-apoptotic protein belonging to the Bcl-2 family. They serve as key regulators at the early stage of apoptosis (27). Our results demonstrated that $\mathrm{I} / \mathrm{R}$ injury increased Bax expression and decreased Bcl-2 expression in the penumbra cortex after cerebral I/R injury. However, our findings further revealed that EPC administration significantly reversed these expression changes, indicating that EPCs may protect neurons from apoptosis. These results are consistent with previous findings showing that peripheral blood or bone marrow-derived EPCs can release various potent inhibitors of apoptosis $(28,29)$. In addition, several reports have also shown that transplantation of EPCs can inhibit cardiac apoptosis in rat models of myocardial infarction (30) and diabetic cardiomyopathy (31). Collectively, our observations demonstrated that EPC implantation is able to prevent neuronal apoptosis induced by I/R injury.

It is well known that a cascade of pathological events, such as oxidative stress, inflammatory responses, neuronal apoptosis and brain damage, occurs within minutes after the onset of cerebral ischemia (4). Enhanced generation of reactive oxygen species (ROS) is considered an important contributor to neuronal damage during ischemic insult. Under normal physiological conditions, the basal amounts of ROS can be rapidly scavenged by a system of endogenous antioxidant defenses, such as SOD, GSH-PX and GSH. However, the relative reduced capability of the antioxidant during cerebral ischemia leads to an excess of ROS production and subsequent neuronal damage (32). The level of MDA, an indicator of oxidative stress, is also increased during cerebral I/R injury. $\mathrm{NF}-\kappa \mathrm{B}$ plays a critical role in regulating cellular responses to oxidative stress and has been shown to be activated during cerebral I/R injury (17). The present study showed that the marked reduction of MDA and $N F-\kappa B$ expression and the significant elevation of SOD, GSH as well as GSH-PX were observed after transplantation of EPCs to ischemia-induced rats. Collectively, the neuroprotective effects of EPC transplantation against brain I/R injury is, at least in part, related to its antioxidant property.

In conclusion, the present study demonstrated that transplantation of bone marrow EPCs exerts potent neuroprotective functions against cerebral I/R injury in rats, and the protective effects may be partly due to its antioxidative and anti-apoptotic properties. Therefore, transplantation of bone marrow EPCs may provide a novel therapeutic strategy for the treatment of ischemic stroke.

\section{References}

1. Feigin VL: Stroke epidemiology in the developing world. Lancet 365: 2160-2161, 2005.

2. Donnan GA, Fisher M, Macleod M and Davis SM: Stroke. Lancet 371: 1612-1623, 2008.

3. Feigin VL, Lawes CM, Bennett DA and Anderson CS: Stroke epidemiology: a review of population-based studies of incidence, prevalence, and case-fatality in the late 20 th century. Lancet Neurol 2: 43-53, 2003.

4. Durukan A and Tatlisumak T: Acute ischemic stroke: overview of major experimental rodent models, pathophysiology, and therapy of focal cerebral ischemia. Pharmacol Biochem Behav 87: 179-197, 2007.

5. Kwiatkowski TG, Libman RB, Frankel M, et al: Effects of tissue plasminogen activator for acute ischemic stroke at one year. National Institute of Neurological Disorders and Stroke Recombinant Tissue Plasminogen Activator Stroke Study Group. N Engl J Med 340: 1781-1787, 1999.

6. Ginsberg MD: Neuroprotection for ischemic stroke: past, present and future. Neuropharmacology 55: 363-389, 2008.

7. Asahara T, Murohara T, Sullivan A, et al: Isolation of putative progenitor endothelial cells for angiogenesis. Science 275: 964-967, 1997.

8. Rafii S and Lyden D: Therapeutic stem and progenitor cell transplantation for organ vascularization and regeneration. Nat Med 9: 702-712, 2003

9. Allegra A, Coppolino G, Bolignano D, et al: Endothelial progenitor cells: pathogenetic role and therapeutic perspectives. J Nephrol 22: 463-475, 2009.

10. Sen S, McDonald SP, Coates PT and Bonder CS: Endothelial progenitor cells: novel biomarker and promising cell therapy for cardiovascular disease. Clin Sci (Lond) 120: 263-283, 2011.

11. Grisar JC, Haddad F, Gomari FA and Wu JC: Endothelial progenitor cells in cardiovascular disease and chronic inflammation: from biomarker to therapeutic agent. Biomark Med 5: 731-744, 2011.

12. Ohta T, Kikuta K, Imamura H, et al: Administration of ex vivoexpanded bone marrow-derived endothelial progenitor cells attenuates focal cerebral ischemia-reperfusion injury in rats. Neurosurgery 59: 679-686, 2006.

13. Rouhl RP, van Oostenbrugge RJ, Damoiseaux J, Tervaert JW and Lodder J: Endothelial progenitor cell research in stroke: a potential shift in pathophysiological and therapeutical concepts. Stroke 39: 2158-2165, 2008.

14. Broughton BR, Reutens DC and Sobey CG: Apoptotic mechanisms after cerebral ischemia. Stroke 40: e331-e339, 2009.

15. Song YS, Narasimhan P, Kim GS, Jung JE, Park EH and Chan PH: The role of Akt signaling in oxidative stress mediates NF-kappaB activation in mild transient focal cerebral ischemia. J Cereb Blood Flow Metab 28: 1917-1926, 2008. 
16. Denk A, Wirth T and Baumann B: NF-kappaB transcription factors: critical regulators of hematopoiesis and neuronal survival. Cytokine Growth Factor Rev 11: 303-320, 2000.

17. Schneider A, Martin-Villalba A, Weih F, Vogel J, Wirth T and Schwaninger M: NF-kappaB is activated and promotes cell death in focal cerebral ischemia. Nat Med 5: 554-559, 1999.

18. Desai A, Singh N and Raghubir R: Neuroprotective potential of the NF-kappaB inhibitor peptide IKK-NBD in cerebral ischemiareperfusion injury. Neurochem Int 57: 876-883, 2010.

19. Zhang SJ, Zhang H, Hou M, et al: Is it possible to obtain 'true endothelial progenitor cells' by in vitro culture of bone marrow mononuclear cells? Stem Cells Dev 16: 683-690, 2007.

20. Timmermans F, Plum J, Yoder MC,IngramDA, Vandekerckhove B and Case J: Endothelial progenitor cells: identity defined? J Cell Mol Med 13: 87-102, 2009.

21. Longa EZ, Weinstein PR, Carlson S and Cummins R: Reversible middle cerebral artery occlusion without craniectomy in rats. Stroke 20: 84-91, 1989.

22. Belayev L, Busto R, Zhao W, Fernandez G and Ginsberg MD: Middle cerebral artery occlusion in the mouse by intraluminal suture coated with poly-L-lysine: neurological and histological validation. Brain Res 833: 181-190, 1999.

23. Hirschi KK, Ingram DA and Yoder MC: Assessing identity, phenotype, and fate of endothelial progenitor cells. Arterioscler Thromb Vasc Biol 28: 1584-1595, 2008.

24. Devanesan AJ,Laughlan KA, Girn HR and Homer-Vanniasinkam S: Endothelial progenitor cells as a therapeutic option in peripheral arterial disease. Eur J Vasc Endovasc Surg 38: 475-481, 2009.
25. Xu Q: Progenitor cells in vascular repair. Curr Opin Lipidol 18 534-539, 2007

26. Li J, Han B, Ma X and Qi S: The effects of propofol on hippocampal caspase-3 and Bcl-2 expression following forebrain ischemia-reperfusion in rats. Brain Res 1356: 11-23, 2010.

27. Jeong HS, Choi HY, Choi TW, et al: Differential regulation of the antiapoptotic action of B-cell lymphoma $2(\mathrm{Bcl}-2)$ and $\mathrm{B}$-cell lymphoma extra long (Bcl-xL) by $\mathrm{c}-J u n \mathrm{~N}$-terminal protein kinase (JNK) 1-involved pathway in neuroglioma cells. Biol Pharm Bull 31: 1686-1690, 2008.

28. Kinnaird T, Stabile E, Burnett MS, et al: Marrow-derived stromal cells express genes encoding a broad spectrum of arteriogenic cytokines and promote in vitro and in vivo arteriogenesis through paracrine mechanisms. Circ Res 94: 678-685, 2004.

29. Urbich C, Aicher A, Heeschen C, et al: Soluble factors released by endothelial progenitor cells promote migration of endothelial cells and cardiac resident progenitor cells. J Mol Cell Cardiol 39: 733-742, 2005

30. Sen S, Merchan J, Dean J, et al: Autologous transplantation of endothelial progenitor cells genetically modified by adenoassociated viral vector delivering insulin-like growth factor-1 gene after myocardial infarction. Hum Gene Ther 21: 1327-1334, 2010.

31. Cheng Y, Guo S, Liu G, et al: Transplantation of bone marrowderived endothelial progenitor cells attenuates myocardial interstitial fibrosis and cardiac dysfunction in streptozotocininduced diabetic rats. Int J Mol Med 30: 870-876, 2012.

32. Allen CL and Bayraktutan U: Oxidative stress and its role in the pathogenesis of ischaemic stroke. Int J Stroke 4: 461-470, 2009. 